\title{
Where the congenital heart disease meets the pulmonary arterial hypertension, FLNA matters: a case report and literature review
}

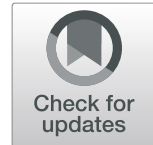

\author{
Xiaoxian Deng ${ }^{1}$, Shanshan $\mathrm{Li}^{1}$, Qiu Qiu', Bowen Jin', Menghuan Yan', Yuanpin $\mathrm{Hu}^{2}$, Yang $\mathrm{Wu}^{3}$, Hongmei Zhou', \\ Gangcheng Zhang ${ }^{1}$ and Xuan Zheng ${ }^{1,2^{*}}$
}

\begin{abstract}
Background: Pediatric patients with genetic disorders have a higher incidence of pulmonary arterial hypertension (PAH) regardless of their heart defects. Filamin A (FLNA) mutation is recently recognized to be associated with pediatric pulmonary disorders, however, the clinical courses of PAH related to the mutation were reported in limited cases. Here, we presented a case and pooled data for better understanding of the correlation between FLNA mutation and pediatric PAH.

Case presentation: The patient was a 8-month-old female with repeated episodes of pneumonia. Physical examination revealed cleft lip, cleft palate and developmental retardation. Imaging examination showed a small atrial septal defect (ASD), central pulmonary artery enlargement, left upper lobe of lung atelectasis, and pulmonary infiltration. Genetic test showed she carried a de novo pathogenic variant of FLNA gene (c.5417-1G > A, p.-). Oral medications didn't slow the progression of PAH in the patient, and she died two years later.

Conclusions: FLNA mutation causes rare but progressive PAH in addition to a wide spectrum of congenital heart disease and other comorbidities in pediatric patients. We highly recommend genetic testing for pediatric patients when suspected with PAH. Given the high mortality in this group, lung transplantation may offer a better outcome.
\end{abstract}

Keywords: Pulmonary arterial hypertension, Congenital heart disease, Filamin A

\section{Background}

Pediatric pulmonary arterial hypertension (PAH) is a rare disease with high mortality. Left-to-right shunting, lung diseases and genetic disorders are most common causes leading to $\mathrm{PAH}$ in children[1]. Filamin A (FLNA) is a $280-\mathrm{kD}$ protein widely expressed in the body and regulating cell shape and migration. Among the broad range of diseases associated with FLNA mutation, lung diseases have been seen in most patients, such as

\footnotetext{
* Correspondence: soniazheng@outlook.com

${ }^{1}$ Congenital Heart Disease center, Wuhan Asia Heart hospital, 753 Jinghan Ave, 430022 Wuhan, China

${ }^{2}$ Laboratory of Molecular Cardiology, Wuhan Asia Heart hospital, 753 Jinghan Avn, 430022 Wuhan, China

Full list of author information is available at the end of the article
}

pneumonia, and respiratory failure. In addition, PAH in pediatric patients with FLNA mutation was fatal despite of their congenital heart disease (CHD), and required early lung transplantation[2]. Here we report a female patient with FLNA mutation, who presented with recurrent pneumonia, arterial septal defect (ASD), mild developmental delay and rapidly progressive $\mathrm{PAH}$.

\section{Case presentation}

An 8-month-old female patient was referred to our center due to severe cough, short of breath, fatigue and fever. The patient had nine episodes of pneumonia and cardiomegaly since she was two-month-old. Physical examination revealed cleft lip, which was surgical repaired when

(C) The Author(s). 2020 Open Access This article is licensed under a Creative Commons Attribution 4.0 International License, which permits use, sharing, adaptation, distribution and reproduction in any medium or format, as long as you give appropriate credit to the original author(s) and the source, provide a link to the Creative Commons licence, and indicate if changes were made. The images or other third party material in this article are included in the article's Creative Commons licence, unless indicated otherwise in a credit line to the material. If material is not included in the article's Creative Commons licence and your intended use is not permitted by statutory regulation or exceeds the permitted use, you will need to obtain permission directly from the copyright holder. To view a copy of this licence, visit http://creativecommons.org/licenses/by/4.0/ The Creative Commons Public Domain Dedication waiver (http://creativecommons.org/publicdomain/zero/1.0/) applies to the data made available in this article, unless otherwise stated in a credit line to the data. 
she was 6 months old, and cleft palate. Her finger oxygen saturation was $94 \%$. Transthoracic echocardiography showed there was a $0.5 \times 0.6 \mathrm{~cm}$ ASD with a $2.4 \mathrm{~cm}$ right atrium. Laboratory test showed NT-proBNP was $963 \mathrm{pg} /$ $\mathrm{ml}$. Some of autoimmune antibodies, including dsDNAantibody, SSA/Ro $60 \mathrm{kD}$ antibody, anti-cardiolipid antibody, and anti- $\beta 2$ GPI antibody, were positive. Erythrocyte sedimentation rate (ESR) and C-reaction protein (CRP) were normal. IgG was slightly elevated at $18.40 \mathrm{~g} / \mathrm{L}$, and C3 was $0.83 \mathrm{~g} / \mathrm{L}$. Significantly increased pulmonary vascular resistance (PVR, $17 \mathrm{WU}$ ) was seen in her first right heart catheterization despite of the slightly increased pulmonary artery pressure (PAP, 38/17/24 $\mathrm{mmHg}$ ). Oral furosemide and antisterone were given since then. She was also suggested to inhale oxygen at home even though she maintained her daily activities without additional requirement of oxygen. The patient was re-hospitalized several times because of recurrent pneumonia and heart failure thereafter. Her finger oxygen saturation dropped to $75 \%$ at lowest, and stayed at $95 \%$ or higher when given nasal catheter oxygen inhalation. Hemodynamic parameters turned worse in the second measurements, where PAP increased along with PVR (PAP, 100/50/67 mmHg; PVR, 42 WU). Further examination included chest computed tomography (CT) scan. CT showed infiltration in upper lobes at both sides (Fig. 1a, b), and lung atelectasis in left upper lobe (Fig. 1b). Pulmonary artery and right atrium were significantly dilated (Fig. 1b, star; d). No thrombosis was seen in pulmonary artery. The patient and her parents received whole exome sequencing test. A new splicing variant (exon34: c.5417-1G > A, p.-) in the FLNA gene was found only in the patient. Diuretics, dopamine, and oral Bosen$\tan$ (12.5 mg twice daily) were used to relieve her symptoms. No intubation or other advanced life supports were required during hospitalizations. Patient's family refused any further intervention during her last hospitalization at age of 2 years. She became significantly cyanosis after last discharge. Unfortunately, the patient didn't response well to medication therapy, and she died from a severe pneumonia 5 months later.

\section{Discussion and conclusions}

$\mathrm{PAH}$ is a clinical symptom characterized by increased pulmonary artery pressure more than $25 \mathrm{mmHg}$. Pediatric PAH shares similarities with adult PAH in some etiology. However, specialists have addressed that pediatric patients have higher prevalence of idiopathic $\mathrm{PAH}, \mathrm{PAH}$ associated with congenital heart disease (CHD), and pulmonary disorders [3]. With the attempt to explore mechanism underlying, next generation sequencing reveals the genetic defects associated with pediatric $\mathrm{PAH}$.

FLNA gene was firstly related to neurologic disorder defect periventricular heterotopia (PVNH) in 1998 [4]. A broad range of diseases were observed with FLNA mutation thereafter, such as otopalatodigital syndrome (OPD) [5], frontometaphyseal dysplasia (FMD) [6], and Melnick-Needles syndrome (MNS) [5], FG syndrome (FGS), chronic idiopathic intestinal pseudoobstruction (CIIP) [7], cardiac valvular disease (CVD) [8], and others.
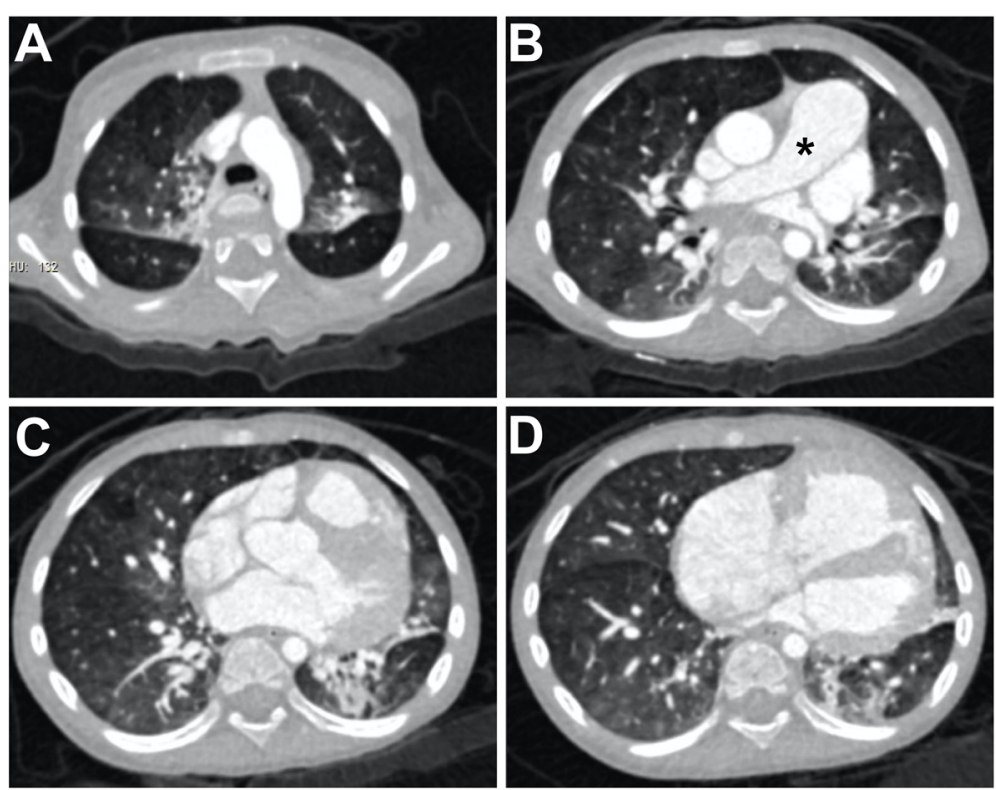

Fig. 1 Chest CT. a Infiltration in both upper lobes of lung; b Main pulmonary artery was dilated $\left(^{*}\right)$. There was atelectasis in left upper lobe of lung; c Slightly infiltration in lower lobes; d Dilated right atrium. 
Table 1 Summary of pediatric PAH associated with FLNA mutation

\begin{tabular}{|c|c|c|c|c|c|c|c|c|}
\hline & Mutation & Sex & $\begin{array}{l}\text { Age at } \\
\text { diagnose }\end{array}$ & CHD & Chest CT & $\begin{array}{l}\text { Lung } \\
\text { transplantation }\end{array}$ & Medicine & Outcome \\
\hline $\begin{array}{l}\text { Masurel- } \\
\text { Paulet } \\
2011[13]\end{array}$ & $\begin{array}{l}\text { mosaic nonsense mutation } \\
\text { c.994delG(p.K331X) }\end{array}$ & male & 3 months & PDA & $\begin{array}{l}\text { Bilateral atelectasis; lung cysts; } \\
\text { tracheobronchomalacia; } \\
\text { pulmonary emphysema; } \\
\text { congenital lobar emphysema; }\end{array}$ & $\mathrm{N}$ & None & ND \\
\hline \multirow[t]{3}{*}{$\begin{array}{l}\text { Reinstein } \\
2013[14]\end{array}$} & $\begin{array}{l}\text { De novo c.2193C > A } \\
\text { (p.Tyr731X) }\end{array}$ & female & 6 months & PDA & $\begin{array}{l}\text { Areas of focal hyperinflation } \\
\text { associated with minimal patchy } \\
\text { atelectasis }\end{array}$ & N & Sildenifil & ND \\
\hline & $\begin{array}{l}\text { De novo deletion of exons } 2,5 \\
\text { and } 13\end{array}$ & female & $\begin{array}{l}18 \\
\text { months }\end{array}$ & VSD & N/A & $\mathrm{N}$ & Bosentan & ND \\
\hline & $\begin{array}{l}\text { De novo } \\
\text { c.5498_- } \\
\text { 5504delCACCCACinsAC }\end{array}$ & male & 2 months & $\begin{array}{l}\text { ASD; } \\
\text { VSD; } \\
\text { PDA }\end{array}$ & N/A & $\mathrm{N}$ & None & Died \\
\hline $\begin{array}{l}\text { Lord } 2014 \\
\text { [12] }\end{array}$ & $\begin{array}{l}\text { Truncating filamin A } \\
\text { mutation(c.5683G-T, p.G1895*) }\end{array}$ & female & 4 months & ASD & $\begin{array}{l}\text { Bilateral pulmonary atelectasis } \\
\text { and cysts, } \\
\text { tracheobronchomalacia, Areas of } \\
\text { hyperinflation alternating with } \\
\text { heterogeneous areas of } \\
\text { atelectasis; alveolar simplification }\end{array}$ & N & $\begin{array}{l}\text { Inhaled } \\
\text { nitric } \\
\text { oxide; } \\
\text { sidenafil; } \\
\text { bosentan }\end{array}$ & ND \\
\hline $\begin{array}{l}\text { Eltahir } 2016 \\
\text { [13] }\end{array}$ & c.3153dupc in exon 21 & female & 2 months & PDA & $\begin{array}{l}\text { Bilateral lung emphysema with } \\
\text { basal atelectasis; bronchospasm }\end{array}$ & N & None & Died \\
\hline \multirow[t]{6}{*}{$\begin{array}{l}\text { Burrage } 2017 \\
{[15]}\end{array}$} & $\begin{array}{l}\text { Heterozygous } \\
\text { c.4596dupG } \\
\text { (p.Ser1533Glufs*12) (de novo) }\end{array}$ & female & 4 months & $\begin{array}{l}\text { PFO, } \\
\text { PDA }\end{array}$ & $\begin{array}{l}\text { Multifocal atelectasis; perinflation } \\
\text { and hyperlucency; atelectasis; } \\
\text { central pulmonary artery } \\
\text { enlargement; } \\
\text { tracheobronchomalacia }\end{array}$ & Y & Sildenafil & Alive \\
\hline & $\begin{array}{l}\text { Heterozygous } \\
\text { c.5290G > A } \\
\text { (p.Ala1764Thr) } \\
\text { (de novo) }\end{array}$ & female & 2 months & $\begin{array}{l}\text { PFO, } \\
\text { PDA, } \\
\text { VSD }\end{array}$ & $\begin{array}{l}\text { Perinflation hyperlucency; } \\
\text { atelectasis; central pulmonary } \\
\text { artery enlargement; } \\
\text { tracheobronchomalacia }\end{array}$ & Y & Sildenafil & Died \\
\hline & $\begin{array}{l}\text { Heterozygous } \\
\text { c.4446_- } \\
\text { 4447dupAT(p.Leu1483Tyrfs* } \\
\text { 19) (de novo) }\end{array}$ & female & 1 month & $\begin{array}{l}\text { PFO, } \\
\text { PDA }\end{array}$ & $\begin{array}{l}\text { Perinflation hyperlucency; } \\
\text { atelectasis; central pulmonary } \\
\text { artery enlargement; } \\
\text { tracheobronchomalacia }\end{array}$ & Y & Sildenafil & Alive \\
\hline & $\begin{array}{l}\text { Heterozygous } \\
\text { c.4617_- } \\
\text { 4618delGC(p.Leu1540Alafs*) }\end{array}$ & female & 2 months & $\begin{array}{l}\text { PFO, } \\
\text { PDA }\end{array}$ & $\begin{array}{l}\text { Perinflation hyperlucency; } \\
\text { atelectasis; central pulmonary } \\
\text { artery enlargement; } \\
\text { tracheobronchomalacia }\end{array}$ & Y & Sildenafil & Alive \\
\hline & $\begin{array}{l}\text { Heterozygous } \\
\text { c.6585dupT } \\
\text { (p.Pro2196Serfs*3) (de novo) }\end{array}$ & female & 7 montsh & $\begin{array}{l}\text { PFO; } \\
\text { PDA }\end{array}$ & $\begin{array}{l}\text { Perinflation hyperlucency; } \\
\text { atelectasis; central pulmonary } \\
\text { artery enlargement; } \\
\text { tracheobronchomalacia }\end{array}$ & Y & Sildenafil & Alive \\
\hline & $\begin{array}{l}\text { Heterozygous } \\
\text { c.2807A > G } \\
\text { (p.Lys936Arg) (VUS) }\end{array}$ & female & 5 months & $\begin{array}{l}\text { PFO, } \\
\text { PDA }\end{array}$ & $\begin{array}{l}\text { Perinflation hyperlucency; } \\
\text { atelectasis; central pulmonary } \\
\text { artery enlargement; } \\
\text { tracheobronchomalacia }\end{array}$ & Y & Sildenafil & Alive \\
\hline \multirow[t]{3}{*}{$\begin{array}{l}\text { Shelmerdine } \\
2017[10]\end{array}$} & $\begin{array}{l}\text { Heterozygous for } \\
\text { c.88delG, p.(Ala30fs) }\end{array}$ & female & ND & $\begin{array}{l}\text { PDA; } \\
\text { PFO }\end{array}$ & $\begin{array}{l}\text { Left lung hyperinflation; } \\
\text { interstitial thickening in left; } \\
\text { mediastinal shift to the right; } \\
\text { right lobe consolidation }\end{array}$ & N & None & Died \\
\hline & $\begin{array}{l}\text { Heterozygous for c.6496dupA, } \\
\text { p. } \\
\text { (lle2166fs) }\end{array}$ & female & ND & PDA & $\begin{array}{l}\text { Progressive right lung } \\
\text { hyperinflation; mediastinal shift } \\
\text { to the left; right upper and } \\
\text { middle lobe over inflation; } \\
\text { coarse septal thickening; lower } \\
\text { lobe atelectasis; patchy ground } \\
\text { glass changes in lower lobes }\end{array}$ & $\mathrm{N}$ & Sildenafil & Alive \\
\hline & $\begin{array}{l}\text { Heterozygous for } \\
\text { c.2190_2193delTTAC, } \\
\text { p(tyr731fs) }\end{array}$ & female & ND & $\begin{array}{l}\text { ASD; } \\
\text { PDA }\end{array}$ & $\begin{array}{l}\text { Right upper lobe hyperinflation; } \\
\text { right middle lobe and left lower } \\
\text { lobe atelectasis; right upper and }\end{array}$ & N & None & Alive \\
\hline
\end{tabular}


Table 1 Summary of pediatric PAH associated with FLNA mutation (Continued)

\begin{tabular}{|c|c|c|c|c|c|c|c|c|}
\hline & Mutation & Sex & $\begin{array}{l}\text { Age at } \\
\text { diagnose }\end{array}$ & CHD & Chest CT & $\begin{array}{l}\text { Lung } \\
\text { transplantation }\end{array}$ & Medicine & Outcome \\
\hline & & & & & $\begin{array}{l}\text { middle, left upper lobe over- } \\
\text { inflation; coarse septal thicken- } \\
\text { ing; lower lobe atelectasis }\end{array}$ & & & \\
\hline $\begin{array}{l}\text { Kinane } 2017 \\
{[16]}\end{array}$ & $\begin{array}{l}\text { c.6577delC; } \\
\text { p.Arg2193AlafsX14[R219AfsX14 }\end{array}$ & female & $7 w$ & $\begin{array}{l}\text { PFO; } \\
\text { VSD; } \\
\text { PDA }\end{array}$ & $\begin{array}{l}\text { Wilson-Mikity syndrome } \\
\text { (pulmonary dysmaturity } \\
\text { syndrome) }\end{array}$ & $\mathrm{N}$ & None & ND \\
\hline $\begin{array}{l}\text { Sasaki } 2018 \\
{[17]}\end{array}$ & Deletion c.6670-1 delG & male & neonate & PDA & $\begin{array}{l}\text { Bilateral dependent and } \\
\text { subsegmental atelectasis, } \\
\text { scattered opacity }\end{array}$ & N & None & Died \\
\hline $\begin{array}{l}\text { Cannaerts } \\
2018[18]\end{array}$ & $\begin{array}{l}\text { cis-located c.7921C > G, } \\
\text { p.Pro2641Ala,c.7923delC, } \\
\text { p.Tyr2642Thrfs*63 }\end{array}$ & female & ND & ASD & ND & N & None & Died \\
\hline This case & $\begin{array}{l}\text { splicing c.5417-1G > A (exon } \\
\text { 34) }\end{array}$ & female & $\begin{array}{l}22 \\
\text { months }\end{array}$ & ASD & $\begin{array}{l}\text { Central pulmonary artery } \\
\text { enlargement; left upper lobe } \\
\text { atelectasis }\end{array}$ & N & Bosentan & Died \\
\hline
\end{tabular}

FLNA Filamin A; CT computed tomography; PDA patent ductus arteriosus; VSD ventricle septal defect; $A S D$ atrial septal defect; $N$ no; $Y$ yes; $N D$ Not provided

However, lung disease was noticed in patients with FLNA mutation first by de Wit MC, et al. in 2010 [9]. Patients with lung disease related to FLNA mutation had higher incidence of pneumonia, lung developmental defects and respiratory failure, however, $\mathrm{PAH}$ were uncommon [10-12]. Among the reported cases, there were 19 of them having early onset PAH (including this case). Their clinical characteristics are summarized in Table 1. Developmental delay was observed in 6 patients, while CHD were seen in all. Fourteen patients had surgical correction of CHD, 6 of which had lung transplantation at the same time. Only one patient died after lung transplantation, nonetheless, mortality among pediatric $\mathrm{PAH}$ patients with FLNA mutation is as high as $35 \%$.

Interstitial lung disease (ILD) may cause $\mathrm{PAH}$ in pediatric patients, and FLNA mutation has been called for attention in ILD [17], but pediatric PAH patients with FLNA mutation don't always present with characteristically pulmonary pathologic changes of ILD. Moreover, high prevalence of CHD in patients with FLNA mutation may confuse the real cause of the rapidly progressive $\mathrm{PAH}$ [19]. In our experience, genetic testing is more helpful to offer early-stage and accurate diagnose. Moreover, lung transplantation would bring higher survival in these patients based on previous reports.

\footnotetext{
Abbreviations

FLNA: Filamin A; CHD: Congenital heart disease; ASD: Atrial septal defect; VSD: Ventricle septal defect; PDA: Patent ductus arteriosus; PFO: Patent foramen ovale; RHC: Right heart catheterization; PAH: Pulmonary arterial hypertension; NT-proBNP: N-terminal prohormone of brain natriuretic peptide; ESR: Erythrocyte sedimentation rate; CRP: C-reaction protein; PVR: Pulmonary vascular resistance; WU: Wood unites; PAP: Pulmonary artery pressure; CT: Computed tomography; PVNH: Periventricular heterotopia; OPD: Otopalatodigital syndrome; FMD: Frontometaphyseal dysplasia; MNS: Melnick-Needles syndrome; FGS: FG syndrome; CIIP: Chronic idiopathic intestinal pseudoobstruction; CVD: Cardiac valvular disease
}

\section{Acknowledgements}

We would like to thank the patient's family for their consent to publish this report. We also appreciated our medical team and their efforts to treat the patient.

\section{Authors' contributions}

$X D, S L, X Z$ management of the patient, drafting the article, critical revision of the article; $Q Q, B J, M Y$ literature review, critical revision of the article; $H$ data collection; YW imaging evaluation; $H Z$, GZ critical revision of the article. All authors read and approved the final manuscript.

\section{Funding}

The authors declare that they did not receive any source of funding for the preparation of the manuscript.

\section{Availability of data and materials}

The datasets used in current study are available from the corresponding author on reasonable request.

Ethics approval and consent to participate

The study was performed according to the Declaration of Helsinki. Written informed consent was obtained from the patient's parents for publication of this case report and accompanying images.

\section{Consent for publication}

Written informed consent was obtained from the patient's parents for publication of this case report and accompanying images.

\section{Competing interests}

The authors declare that they have no competing interests.

\section{Author details}

${ }^{1}$ Congenital Heart Disease center, Wuhan Asia Heart hospital, 753 Jinghan Ave, 430022 Wuhan, China. ${ }^{2}$ Laboratory of Molecular Cardiology, Wuhan Asia Heart hospital, 753 Jinghan Avn, 430022 Wuhan, China. ${ }^{3}$ Imaging center, Wuhan Asia Heart hospital, 753 Jinghan Ave, 430022 Wuhan, China.

Received: 2 October 2019 Accepted: 15 October 2020

Published online: 03 November 2020

\section{References}

1. Morrell NW, Aldred MA, Chung WK, Elliott CG, Nichols WC, Soubrier F, Trembath RC, Loyd JE. Genetics and genomics of pulmonary arterial hypertension. Eur Respir J. 2019;53(1).

2. Pelizzo G, Collura M, Puglisi A, Pappalardo MP, Agolini E, Novelli A, Piccione M, Cacace C, Bussani R, Corsello G, et al. Congenital emphysematous lung 
disease associated with a novel Filamin A mutation. Case report and literature review. BMC Pediatr. 2019;19(1):86.

3. Rosenzweig EB, Abman SH, Adatia I, Beghetti M, Bonnet D, Haworth S, Ivy DD, Berger RMF. Paediatric pulmonary arterial hypertension: updates on definition, classification, diagnostics and management. Eur Respir J. 2019; 53(1).

4. Fox JW, Lamperti ED, Eksioglu YZ, Hong SE, Feng Y, Graham DA, Scheffer IE, Dobyns WB, Hirsch BA, Radtke RA, et al. Mutations in filamin 1 prevent migration of cerebral cortical neurons in human periventricular heterotopia. Neuron. 1998;21(6):1315-25.

5. Robertson SP, Twigg SR, Sutherland-Smith AJ, Biancalana V, Gorlin RJ, Horn D, Kenwrick SJ, Kim CA, Morava E, Newbury-Ecob R, et al. Localized mutations in the gene encoding the cytoskeletal protein filamin A cause diverse malformations in humans. Nat Genet. 2003;33(4):487-91.

6. Zenker M, Nahrlich L, Sticht H, Reis A, Horn D. Genotype-epigenotypephenotype correlations in females with frontometaphyseal dysplasia. Am J Med Genet A. 2006;140(10):1069-73.

7. Gargiulo A, Auricchio R, Barone MV, Cotugno G, Reardon W, Milla PJ Ballabio A, Ciccodicola A, Auricchio A. Filamin A is mutated in X-linked chronic idiopathic intestinal pseudo-obstruction with central nervous system involvement. Am J Hum Genet. 2007;80(4):751-8.

8. Kyndt F, Gueffet JP, Probst V, Jaafar P, Legendre A, Le Bouffant F, Toquet C, Roy E, McGregor L, Lynch SA, et al. Mutations in the gene encoding filamin A as a cause for familial cardiac valvular dystrophy. Circulation. 2007;115(1): 40-9.

9. de Wit MC, Tiddens HA, de Coo IF, Mancini GM. Lung disease in FLNA mutation: confirmatory report. Eur J Med Genet. 2011;54(3):299-300.

10. Shelmerdine SC, Semple T, Wallis C, Aurora P, Moledina S, Ashworth MT, Owens CM. Filamin A (FLNA) mutation-A newcomer to the childhood interstitial lung disease (ChILD) classification. Pediatr Pulmonol. 2017;52(10): 1306-15.

11. Eltahir S, Ahmad KS, Al-Balawi MM, Bukhamsien H, Al-Mobaireek K, Alotaibi W, Al-Shamrani A. Lung disease associated with filamin A gene mutation: a case report. J Med Case Rep. 2016;10:97.

12. Lord A, Shapiro AJ, Saint-Martin C, Claveau M, Melancon S, Wintermark P. Filamin A mutation may be associated with diffuse lung disease mimicking bronchopulmonary dysplasia in premature newborns. Respir Care. 2014; 59(11):e171-7.

13. Masurel-Paulet A, Haan E, Thompson EM, Goizet C, Thauvin-Robinet C, Tai A, Kennedy D, Smith G, Khong TY, Sole G, et al. Lung disease associated with periventricular nodular heterotopia and an FLNA mutation. Eur I Med Genet. 2011;54(1):25-8.

14. Reinstein E, Frentz S, Morgan T, Garcia-Minaur S, Leventer RJ, McGillivray G, Pariani M, van der Steen A, Pope M, Holder-Espinasse M, et al. Vascular and connective tissue anomalies associated with X-linked periventricular heterotopia due to mutations in Filamin A. Eur J Hum Genet. 2013;21(5): 494-502.

15. Burrage LC, Guillerman RP, Das S, Singh S, Schady DA, Morris SA, Walkiewicz M, Schecter MG, Heinle JS, Lotze TE, et al. Lung transplantation for FLNAassociated progressive lung disease. J Pediatr. 2017:186:118-123 e116.

16. Kinane TB, Lin AE, Lahoud-Rahme M, Westra SJ, Mark EJ. Case 4-2017. A 2month-old girl with growth retardation and respiratory failure. $\mathrm{N} \mathrm{Engl} \mathrm{J}$ Med. 2017;376(6):562-74.

17. Sasaki E, Byrne AT, Phelan E, Cox DW, Reardon W. A review of filamin A mutations and associated interstitial lung disease. Eur J Pediatr. 2019;178(2): $121-9$.

18. Cannaerts E, Shukla A, Hasanhodzic M, Alaerts M, Schepers D, Van Laer L, Girisha KM, Hojsak I, Loeys B, Verstraeten A. FLNA mutations in surviving males presenting with connective tissue findings: two new case reports and review of the literature. BMC Med Genet. 2018;19(1):140.

19. Demirel N, Ochoa R, Dishop MK, Holm T, Gershan W, Brottman G. Respiratory distress in a 2-month-old infant: is the primary cause cardiac, pulmonary or both? Respir Med Case Rep. 2018;25:61-5.

\section{Publisher's Note}

Springer Nature remains neutral with regard to jurisdictional claims in published maps and institutional affiliations.

Ready to submit your research? Choose BMC and benefit from:

- fast, convenient online submission

- thorough peer review by experienced researchers in your field

- rapid publication on acceptance

- support for research data, including large and complex data types

- gold Open Access which fosters wider collaboration and increased citations

- maximum visibility for your research: over $100 \mathrm{M}$ website views per year

At BMC, research is always in progress.

Learn more biomedcentral.com/submissions 